\title{
Optical biopsy using tissue spectroscopy and optical coherence tomography
}

\author{
Norman S Nishioka MD
}

NS Nishioka. Optical biopsy using tissue spectroscopy and optical coherence tomography. Can J Gastroenterol 2003;17(6):376-380.

\begin{abstract}
'Optical biopsy' or 'optical diagnostics' is a technique whereby light energy is used to obtain information about the structure and function of tissues without disrupting them. In fluorescence spectroscopy, light energy (usually provided by a laser) is used to excite tissues and the resulting fluorescence provides information about the target tissue. Its major gastrointestinal application has been in the evaluation of colonic polyps, in which it can reliably distinguish malignant from benign lesions. Optical coherence tomography (OCT) has been used in the investigation of Barrett's epithelium (and dysplasia), although a variety of other applications are feasible. For example, OCT could assist in the identification and staging of mucosal and submucosal neoplasms, the grading of inflammation in the stomach and intestine, the diagnosis of biliary tumours and the assessment of villous architecture. OCT differs from endoscopic ultrasound, a complementary modality, in that it has a much higher resolution but lesser depth of penetration. The images correlate with the histopathological appearance of tissues, and the addition of Doppler methods may enable it to evaluate the vascularity of tumours and the amount of blood flow in varices. Refinements in these new optical techniques will likely make them valuable in clinical practice, although their specific roles have yet to be determined.
\end{abstract}

Key Words: Laser; Optical coherence tomography; Optics; Spectroscopy

\section{La biopsie optique au moyen de la spectro- scopie tissulaire et de la tomographie par cohérence optique}

\begin{abstract}
La «biopsie optique », ou « diagnostic optique », est une technique selon laquelle l'énergie lumineuse est utilisée pour obtenir de l'information sur la structure et la fonction des tissus sans les perturber. En cas de spectroscopie par fluorescence, l'énergie lumineuse (souvent fournie par laser) est utilisée pour exciter les tissus, et la fluorescence résultante fournit de l'information au sujet des tissus cibles. Sa principale application en gastroentérologie est l'évaluation des polypes coliques, car elle permet de distinguer avec fiabilité les lésions malignes des lésions bénignes. La tomographie par cohérence optique (TCO) est utilisée dans l'exploration de l'épithélium de Barrett (et de la dysplasie), bien que diverses autres applications soient possibles. Par exemple, la TCO pourrait contribuer au dépistage et à la classification des néoplasmes muqueux et sous-muqueux, à la gradation de l'inflammation dans l'estomac et l'intestin, au diagnostic des tumeurs biliaires et à l'évaluation de l'architecture villeuse. La TCO diffère de l'échographie endoscopique, une modalité complémentaire, parce que sa résolution est beaucoup plus élevée, mais sa profondeur de pénétration moindre. Les images sont mises en corrélation avec l'apparence histopathologique des tissus, et l'ajout de méthodes de Doppler peut lui permettre d'évaluer la vascularité des tumeurs et l'importance du débit sanguin dans les varices. Les raffinements de ces nouvelles techniques optiques les rendront probablement précieuses en pratique clinique, même si leurs rôles précis ne sont pas encore déterminés.
\end{abstract}

\section{BACKGROUND}

The term 'optical biopsy' usually refers to a technique by which an optical device is used to render a tissue diagnosis in situ. The term is somewhat of a misnomer because the term 'biopsy' implies that tissue is removed, whereas one of the goals of this technology is to reduce or even eliminate the need for an actual biopsy. An alternative and more accurate term that is becoming increasing popular is 'optical diagnostics'. Diagnostic information is obtained by using light, without tissue disruption or destruction.

The gastrointestinal (GI) tract is well suited to the application of optical diagnostic methods during endoscopy. Although a wide variety of optical techniques may be used, the majority of techniques are based on conventional laboratory spectroscopic methods, such as diffuse reflectance spec- troscopy, fluorescence spectroscopy, and Raman scattering. Numerous clinical applications of these modalities have been explored, including distinguishing malignant or dysplastic tissue from benign tissue, high-resolution imaging, monitoring the metabolic state and measuring local drug concentrations.

Because optical diagnostic strategies frequently use visible and near-infrared light to probe tissue, most can use small diameter optical fibres. This makes many optical diagnostic techniques easy to apply to endoscopic procedures, whereby the devices can either be passed through the instrument channels of endoscopes or be integrated into the fiberoptic viewing bundles of nonvideo instruments. These techniques, therefore, enhance the ability of the endoscopist to evaluate the nature and state of GI tissues. Fluorescence spectroscopy and optical coherence tomography (OCT) have generated the most recent interest.

This article was originally presented at the 15th International Course on Therapeutic Endoscopy, hosted by the Wellesley Group, Division of Gastroenterology, St Michael's Hospital, Toronto, Ontario, October 9-12, 2002

Massachusetts General Hospital, Boston, Massachusetts, USA

Correspondence: Dr Norman S Nishioka, Massachusetts General Hospital, Blake 4, 55 Fruit Street, Boston, Massachusetts 02114, USA.

Telephone 617-726-8409, fax 617-726-4103, e-mail norm@wlp.mgh.harvard.edu 


\section{FLUORESCENCE SPECTROSCOPY}

Fluorescence spectroscopy is a powerful laboratory technique that is widely used in the fields of chemistry, physics, biochemistry and biophysics to characterize chemical and physical processes in materials. The fluorescence phenomenon involves the absorption of incident light, typically at ultraviolet or visible wavelengths, by an atom, molecule or molecular aggregate. The target substance's energy is increased and fluorescence occurs when the target gives off a portion of this extra energy as fluorescent light. The relationship between wavelength (colour) and intensity of the emitted fluorescence provides an optical 'fingerprint' of the target substance. If the intensity of the fluorescence signal is measured as a function of its wavelength while the excitation wavelength is fixed, the result is an emission spectrum. Conversely, if the emission intensity is monitored at a fixed wavelength while the excitation wavelength is varied, the result is an excitation spectrum.

The compound that produces the fluorescence signal is known as a 'fluorophore'. The term 'autofluorescence' connotes fluorescence that is produced by endogenous tissue fluorophores and is used to distinguish it from the more generic term 'fluorescence' that includes fluorescence from exogenously administered compounds. Laser-induced fluorescence refers to that produced in response to illumination with laser light. Although lasers are not required to make a fluorescence measurement, their monochromatic (single wavelength) nature and high intensity make them convenient light sources for fluorescence spectroscopy.

\section{Instrumentation}

A wide variety of instruments have been employed to acquire fluorescence signals in vivo. The basic elements of a typical system are:

1. An excitation light source provides the incident light for fluorescence. Lasers or filtered lamps are the most frequently used sources;

2. A fiberoptic device is often used for delivering the excitation light to the tissue, and is usually inserted through the instrument channel of an endoscope. Alternatively, the illumination bundles contained within the endoscope itself can be used for this purpose;

3. The resultant fluorescent light can be captured by the fibre that is used to transmit the excitation light, or by an imaging device, such as an endoscope;

4. An optical analyzer module usually determines the fluorescence intensity as a function of wavelength. There are many ways to analyze the optical signals, ranging from the naked eye and simple filters and optical detectors to sophisticated optical multichannel analyzer systems; and

5. The information contained in the fluorescence signal is usually transferred to a computer that performs an analysis algorithm. Not all systems use computers, however, and sometimes the human brain is all that is needed.

\section{Autofluorescence for tissue characterization}

Because fluorescence spectroscopy is used routinely to probe the composition and physiological state of systems in vitro, it is reasonable to hypothesize that tissue autofluorescence could be useful as a nondestructive in vivo tissue probe. During the past 15 years, laser-induced autofluorescence has been examined in a wide variety of tissues (1-5)

In the GI tract, the most thoroughly evaluated application has been the identification of polyp type $(6-8)$. This is an interesting area for research, not only because it is an important clinical problem, but also because it may provide insight into how to identify neoplasia and dysplasia. Several studies from independent research groups, using somewhat different laser excitation wavelengths and discrimination algorithms, have demonstrated positive predictive values of approximately $90 \%$ to $95 \%$ and negative predictive values in the range of $80 \%$ to $90 \%$ for malignant tissue $(9-12)$, that are comparable with those obtained using clinical pathology methods. A commercial device for this application has been approved by the US Food and Drug Administration.

\section{OPTICAL COHERENCE TOMOGRAPHY}

Optical coherence tomography is an optical imaging technique that is analogous to ultrasonography, but uses light instead of an acoustic signal. Cross-sectional images of tissue obtained using current OCT technology have resolutions of roughly $10 \mu \mathrm{m}$, and resolutions as small as $1 \mu \mathrm{m}$ have been demonstrated using laboratory prototypes. Moreover, OCT is an attractive technology because it can be implemented using narrow diameter optical fibres that can be passed through endoscopes.

All images produced by standard endoscopes are necessarily limited to examination of mucosal surfaces. Therefore, essentially no information about the depth of an object or its threedimensional characteristics is available during routine endoscopy. Endoscopic ultrasound (EUS) has greatly enhanced the ability to estimate the depth of lesions and has been used to examine structures in the pancreas, liver or lymph nodes (13). Standard EUS instruments operate at a frequency of $7.5 \mathrm{MHz}$, with a corresponding resolution of approximately $340 \mu \mathrm{m}$ (in water). This relatively low operating frequency permits good depth of penetration into tissue, and structures many centimeters beneath the probe are readily visualized. Higher-frequency ultrasound probes are available but not widely used. These instruments have operating frequencies as great as $25 \mathrm{MHz}$ and can provide resolutions as small as $110 \mu \mathrm{m}$ in the axial direction and $240 \mu \mathrm{m}$ in the lateral direction. OCT provides higher spatial resolution than EUS but a lesser depth of penetration $(14,15)$. Using current technology, OCT and EUS are complementary imaging modalities, which means that OCT is unlikely to totally replace EUS as a routine imaging procedure.

From an operational standpoint, OCT has two characteristics that could make it easier to use and interpret than EUS (15). First, EUS requires that an acoustic match between the transducer and tissue be present, which generally means that water must be interposed between the instrument and the tissue surface. This can be done by operating in a pool of water or in a setting in which water is flowing, or by wrapping a waterfilled balloon around the transducer. In contrast, OCT can operate with either a water or an air interface, which makes it technically simpler to use.

The second difference between OCT and EUS involves the interpretation of images (15). Ultrasound images are difficult for untrained gastroenterologists to interpret, as they are not 
accustomed to viewing images that are based on differences in acoustic impedance. A significant learning curve, therefore, exists for EUS and most practitioners have taken special training in its use. On the other hand, images provided by OCT closely mimic histopathology and, as spatial resolution improves, this match will be enhanced. Because most gastroenterologists have at least passing familiarity with the histopathological appearance of structures in the GI tract, it is possible that they will be able to interpret OCT images more easily than those obtained at EUS. The attributes of OCT and EUS are compared in Table 1.

\section{In vitro studies}

There have been several reports of OCT imaging of in vitro specimens (14-18). Although the results of these studies are of limited clinical relevance, they form the basis for the present understanding of the appearance of GI tissues when using this modality. For example, Izatt and colleagues (14) used a mechanically scanned fibre-based OCT device to obtain images of porcine esophagi in vitro. This system was not designed for clinical use and requires 100 seconds to acquire a single image. In the esophagus, the device was able to distinguish three distinct layers, based on the correlation between the measured thickness of the layers and similar measurements for fixed histological sections. These layers were felt to represent the squamous epithelium, lamina propria and muscularis mucosae. The squamous epithelium was seen as a highly scattering layer, whereas the lamina propria exhibited much less backscatter.

In vivo OCT studies by Bouma and colleagues (19) and by Sergeev and colleagues (20) showed a different backscatter pattern, in that the lamina propria showed a much higher degree of scattering than did the squamous epithelium. In the in vitro study, the submucosa and muscularis propria were poorly visualized, due to attenuation of the incident light beam below detectable levels (14). In that study, OCT imaging was also performed in vitro on a segment of the stomach from a patient with gastric cancer. The normal stomach gave a similar appearance to that of the esophagus, and the mucosa and lamina propria were readily identified. Areas of the stomach involved with adenocarcinoma exhibited increased backscatter. In a sample of normal human colon, the mucosa, muscularis mucosae and submucosa could be distinguished. Interestingly, colonic crypts that were readily seen in histological sections were not resolved by OCT. Although this imaging system was not suitable for clinical use, this study demonstrated the potential value of OCT to provide images of the superficial layers of the GI tract.

Tearney and coworkers (21) reported on an in vitro experience using autopsy specimens. The fiberoptic sample arm of an OCT device was mechanically scanned across the tissue during an acquisition period of $45 \mathrm{~s}$. This system was also not suitable for clinical use. Postmortem images of the esophagus were similar to those obtained by Izatt's team (14). The upper portion of the mucosa appeared homogenous in the OCT images. In these experiments, the muscularis mucosae was more reflective than the mucosa. Colonic inflammation was characterized by an area of high backscatter, and disruption of the crypt architecture was also evident.

In another study, the same investigators examined OCT images of pancreatic and biliary ducts obtained at autopsy (22). They found that OCT was able to distinguish the various layers of the common bile duct. In addition, the glands of the
TABLE 1

\begin{tabular}{lcc}
\multicolumn{3}{l}{ Comparison of optical coherence tomography (OCT) and } \\
conventional endoscopic ultrasonography (EUS) \\
\hline \multicolumn{3}{l}{ OCT } \\
\hline Typical configuration & catheter & EUS \\
Penetration depth & $2 \mathrm{~mm}$ & $5 \mathrm{~cm}$ \\
Spatial resolution (in water) & $7 \mu \mathrm{m}$ & $110 \mu \mathrm{m}(25 \mathrm{MHz})$ \\
Image analogue & histopathology & ultrasound \\
Coupling medium & air or water & water \\
Doppler application & possible & available \\
\hline
\end{tabular}

ducts and their associated ductules could be identified. Images of the pancreatic duct were able to resolve the ductal epithelium, periductal pancreatic tissue and islet cells.

\section{In vivo pilot studies in humans}

The first in vivo demonstration of the human GI tract by OCT was by Sergeev and colleagues in 1997 (20). This was a description of preliminary experience with four patients who underwent gastroscopy. The authors were able to distinguish five layers within the normal esophagus, that they tentatively identified as the mucosa (squamous epithelium), lamina propria, muscularis mucosae, submucosa and muscularis propria. Their assessment was based on an approximate correlation with the thickness of these layers in histological sections, but the relevant biopsy materials were not provided in their paper. Furthermore, the biopsy specimens would not have included all the layers visualized at OCT, and, thus, would not have been able to definitively identify these layers. The authors obtained similar results in the gastric antrum, and stated that OCT could identify the mucosa (columnar epithelium), lamina propria and muscularis mucosae. Other features that may have been resolved included glands, gastric pits and blood vessels. OCT imaging of an esophageal cancer and a gastric cancer revealed an abnormally large number of blood vessels within the tumour mass, suggesting the presence of increased vascularity. In addition, increased backscatter was observed from the epithelial layer of the tumours, which may have reflected greater nuclear density. The same group later published a larger clinical series.

Two other groups have since described the use of OCT in larger numbers of GI patients $(19,23)$. Sivak and colleagues (23) reported their initial experience with 38 patients. Images of normal tissue were obtained from the esophagus, stomach, duodenum, terminal ileum and colon. The normal esophagus exhibited five layers. Structures that were thought to represent glands and blood vessels were seen in the fourth layer, that was thus felt to be the submucosa. This assignment of layers agreed with that reported by the Hamburg team $(20,24)$. Gastric pits and duodenal villi could be identified in the stomach and small intestine, respectively. This study also demonstrated that a probe with a $1-\mathrm{mm}$ focal point seemed to provide optimal image quality. The author's group at the Massachusetts General Hospital has reported the results of OCT in 32 patients (19). This experience has now increased to more than 300 patients.

\section{Potential clinical applications}

To date, the majority of reports of the clinical use OCT have been preliminary survey studies involving only a small number of patients with abnormal tissue. Thus, there are no clearly 
established indications for OCT in clinical gastroenterology. Nevertheless, the experience has been sufficient to suggest possible applications of this technique. Although fine cellular detail is beyond the resolution of current OCT technology, information about tissue architecture is readily apparent in the images.

Barrett's esophagus: In this condition, the normal squamous epithelium of the esophagus is replaced by columnar epithelium. Although three types of Barrett's epithelium have been described historically, only the specialized columnar epithelium (or intestinal metaplasia) is of clinical importance, because of its malignant potential. Therefore, the presence of intestinal metaplasia in the esophagus has become synonymous with Barrett's epithelium.

In a study of 121 patients, the author's group showed that OCT could reliably identify Barrett's epithelium (25). The laminar appearance of the normal squamous mucosa contrasts quite obviously with the columnar architecture of Barrett's mucosa. In addition, the latter exhibits OCT features that readily distinguish it from the normal gastric mucosa. Specifically, OCT images of the gastric mucosa show a small penetration depth, a regular 'crypt-and-pit' architecture and a vertical tissue orientation. In contrast, with intestinal metaplasia, the penetration depth is greater, the regular 'crypt-and-pit' appearance is lost, and the mucosal surface is usually very irregular. In most cases of Barrett's epithelium, OCT reveals mucosal glands that are seen either as pockets of reflectance below the epithelial surface or as invaginations through the epithelium. It is generally not possible to visualize structures deeper than the muscularis mucosae due to loss of the signal or shadowing arising from strong scattering within the metaplastic epithelium. In addition, the distinct tissue layers seen in squamous epithelium are typically obscured in Barrett's epithelium.

Identification of Barrett's esophagus is not usually difficult for the endoscopist, becauses the pearly white squamous mucosa contrasts with the pinker salmon colour of columnar epithelium. In cases of short-segment Barrett's esophagus, however, the squamocolumnar junction may not be obvious endoscopically, and OCT may be valuable.

Dysplasia is identified by cytological and architectural changes. The former, including nuclear size and shape, nuclear/cytoplasmic ratio and nuclear stratification, are beyond the resolution of OCT. Certain subcellular changes, such as increased nuclear/cytoplasmic ratio, however, might alter the light reflection characteristics of Barrett's epithelium, thereby leading to enhanced contrast in OCT images. Preliminary evidence supports the idea that dysplastic Barrett's epithelium could be identified by OCT (26). Moreover, the author's group has observed what may be specific changes in the glandular architecture (eg, irregular shapes and cribriform glands) in cases of high-grade dysplasia. Larger trials will be necessary to determine the sensitivity and specificity of these features for diagnosing high-grade dysplasia.

Gastritis: Gastritis is a generic term that denotes the presence of inflammation within the gastric mucosa. It is a very prevalent condition that can be caused by medications, infection, trauma, excessive gastric acidity or autoimmune disease. Endoscopic findings include erythema that is typically patchy in distribution. Active gastritis can be differentiated from normal gastric mucosa at OCT by the findings of increased backscattering from the mucosal layer and more pronounced 'crypt-and-pit' architecture (27). Although OCT appears to be capable of identifying areas of gastritis, its clinical value in this setting is unclear.
Celiac disease: The villous architecture of the duodenum is readily apparent in OCT images, which means that this technique might be of value in screening patients for diseases like celiac disease that are characterized by villous atrophy. Normal findings at OCT might make biopsies unnecessary. Conversely, since villous atrophy is not a specific abnormality, it is unlikely that OCT will obviate the need to obtain biopsies in such cases.

Inflammatory bowel disease: Mucosal inflammation, such as that seen with inflammatory bowel disease, can generally be identified endoscopically. Endoscopy can provide only a qualitative estimate of the degree of inflammation, however, and OCT may be of value, especially in cases of ulcerative colitis, because it can measure the thickness of the mucosa.

Cancer staging: The majority of GI cancers arise from the mucosa, which makes detection and staging of the disease by OCT feasible and attractive. The tumour can usually be easily seen at endoscopy, but the depth of invasion, which is important to the prognosis and management of the disease, is not readily discernible. Cancer staging is currently most frequently undertaken by EUS. Although EUS can accurately evaluate most cancers, superficial tumours can be difficult to assess. Accurate staging is crucial for these lesions, since some tumours might be successfully managed with local treatment modalities, such as endoscopic mucosectomy and photodynamic therapy. OCT may ultimately prove valuable as a means of selecting patients who would benefit from endoscopic therapy. After treatment, OCT may be able to assess the resection margins to ensure that they are free of tumour.

Submucosal lesions: Although the majority of clinically significant GI abnormalities originate in the mucosa, several types of lesions occur in deeper tissue layers and could be evaluated by OCT. For example, this technique can identify and locate varices and determine their size and depth. The addition of Doppler methods could allow quantification of blood flow $(27,28)$. This information could help determine the prognosis and guide the management of esophageal and gastric varices.

The submucosal and muscular layers of the intestinal wall are often the sites of benign tumours. OCT may allow the endoscopist to identify submucosal lipomas, which do not require resection. Leiomyomas appear endoscopically as smooth mass lesions, and EUS cannot reliably distinguish a benign leiomyoma from its malignant counterpart (leiomyosarcoma). OCT might be of value in making this distinction, especially if its depth of penetration improves, although the concept has not yet been tested in practice.

Pancreatic and biliary ducts: Preliminary experience in acquiring OCT images of the common bile duct has been promising (22). Cholangiocarcinomas can be readily identified and distinguished from benign biliary strictures. It is not clear, however, whether OCT could provide information that cannot be obtained from EUS, endoscopic retrograde cholangiopancreatography or cholangiopancreatoscopy.

\section{FUTURE DIRECTIONS}

Current OCT systems are far from optimal for use in the GI tract. They have been designed using a highly empirical approach. Educated guesses, based on optical properties of various tissues, have been used to select operating parameters. For example, the optimal wavelength for imaging the GI tract is not known. In general, near-infrared wavelengths have been employed because they penetrate more deeply into tissues than 
do shorter wavelengths of light, and because suitable lowcoherence light sources are available. Furthermore, in vitro studies have suggested that varying the wavelength produces only modest changes in image quality. Nevertheless, it is conceivable that the performance characteristics of OCT might be significantly enhanced were different wavelengths of light used. With the availability of newer light sources, it will likely be possible to resolve fine details of cellular architecture. Similarly, available OCT systems employ catheters that vary in their optical properties, and the optimal design for any given clinical application is not yet known. It is likely that no single approach would be best for all endoscopic applications.

For many years, endoscopists have routinely used a variety of dyes to enhance the visualization of mucosal lesions. It is reasonable to assume that dyes could also be helpful during OCT, but essentially nothing is known about their potential usefulness. Since any of a large number of dyes might improve the sensitivity and/or specificity of OCT, this is an area of investigation that offers significant promise.

Doppler techniques have been used with OCT systems $(27,28)$, but not yet in the evaluation of the human GI tract. It can be expected that Doppler technology would provide clini- cally useful information about blood flow and perfusion in the GI tract.

From a clinical perspective, the use of endoscopic OCT in the GI tract is still in its infancy. The feasibility of OCT imaging during routine GI endoscopy has been conclusively demonstrated, and OCT provides the highest resolution of any available technology. As with any emerging technology, it is not yet clear in which clinical situations OCT will prove to be cost effective. Carefully conducted prospective trials will be required to establish the clinical utility of this modality. In the current health care environment of cost awareness, such studies will be crucial to the acceptance of OCT as a routine clinical tool.

\section{CONCLUSIONS}

Are optical diagnostic tools ready for routine clinical use? The overall experience with several different systems has been very encouraging. As the techniques become more refined and endoscopists become more familiar with them, it is very likely that some forms of fluorescence spectroscopy and/or OCT will find important clinical roles. It is also likely that, as they become commercially available, some devices will find their way into common use in the near future.

\section{REFERENCES}

1. Alfano RR, Tata DB, Cordero J, et al. Laser induced fluorescence spectroscopy from native cancerous and normal tissue. IEEE J Quantum Electronics 1984;20:1507-11.

2. Anderson PS, Gustafson A, Stenram U, Svanberg K, Svanberg S. Diagnosis of arterial atherosclerosis using laser-induced fluorescence. Lasers Med Sci 1987;2:261-6.

3. Tralau CJ, Barr H, Sandeman DR, Barton T, Lewin MR, Bown SG. Aluminum sulfonated phthalocyanine distribution in rodent tumors of the colon, brain and pancreas. Photochem Photobiol 1987;46:777-81.

4. Yang YL, Ye YM, Li FM, Li YF, Ma PZ. Characteristic autofluorescence for cancer diagnosis and its origin. Lasers Surg Med 1987;7:528-32

5. Baraga JJ, Feld MS, Rava RP. In situ optical histochemistry of human artery using near infrared Fourier transform Raman spectroscopy. Proc Natl Acad Sci USA 1992;89:3473-7.

6. Yashke PN, Bonner RF, Cohen P, Leon MB, Fleischer DE. Laserinduced fluorescence spectroscopy may distinguish colon cancer from normal human colon. Gastrointestinal Endoscopy 1989;35:184. (Abst)

7. Richards-Kortum R, Rava RP, Petras RE, Fitzmaurice M, Sivak M, Feld MS. Spectroscopic diagnosis of colonic dysplasia. Photochem Photobiol 1991;53:777-86.

8. Schomacker KT, Frisoli JK, Compton CC, et al. Ultraviolet laserinduced fluorescence of colonic tissue: Basic biology and diagnostic potential. Lasers Surg Med 1992;12:63-78.

9. Kapadia CR, Cutruzzola FW, O’Brien KM, Stetz ML, Enriquez R, Deckelbaum LI. Laser-induced fluorescence spectroscopy of human colonic mucosa. Detection of adenomatous transformation. Gastroenterology 1990;99:150-7.

10. Cothren RM, Richards-Kortum R, Sivak MV Jr, et al. Gastrointestinal tissue diagnosis by laser-induced fluorescence spectroscopy at endoscopy. Gastrointest Endosc 1990;36:105-11.

11. Marchesini R, Brambilla M, Pignoli E, et al. Light-induced fluorescence spectroscopy of adenomas, adenocarcinomas and nonneoplastic mucosa in human colon. I. In vitro measurements. J Photochem Photobiol Biol 1992;14:219-30.

12. Schomacker KT, Frisoli JK, Compton CC, et al. Ultraviolet laserinduced fluorescence of colonic polyps. Gastroenterology 1992;102:1155-60

13. Wiersema MJ, Wiersema LM. High-resolution 25-megahertz ultrasonography of the gastrointestinal wall: Histologic correlates. Gastrointest Endosc 1993;39:499-504.

14. Izatt JA, Kulkarni MD, Wang H-W, Kobayashi K, Sivak MV Jr. Optical coherence tomography in gastrointestinal tissues. IEEE J Sel Top Quantum Electronics 1996;2:1017-28.

15. Das A, Sivak MV Jr, Chak A, et al. High-resolution endoscopic imaging of the GI tract: A comparative study of optical coherence tomography versus high-frequency catheter probe EUS. Gastrointest Endosc 2001;54:219-24.

16. Brezinski ME, Tearney GJ, Bouma BE, et al. Optical coherence tomography for optical biopsy. Properties and demonstration of vascular pathology. Circulation 1996;93:1206-13.

17. Brezinski ME, Tearney GJ, Weissman NJ, et al. Assessing atherosclerotic plaque morphology: Comparison of optical coherence tomography and high frequency intravascular ultrasound. Heart 1997;77:397-403.

18. Kobayashi K, Izatt JA, Kulkarni MD, Willis J, Sivak MV Jr. Highresolution cross-sectional imaging of the gastrointestinal tract using optical coherence tomography: Preliminary results. Gastrointest Endosc 1998;47:515-23.

19. Bouma BE, Tearney GJ, Compton CC, Nishioka NS. High-resolution imaging of the human esophagus and stomach in vivo using optical coherence tomography. Gastrointest Endosc 2000;51:467-74.

20. Sergeev AM, Gelikonov VM, Gelikonov GV, et al. In vivo endoscopic OCT imaging of precancer and cancer states of human mucosa. Optics Express 1997;1:432-40.

21. Tearney GJ, Brezinski ME, Southern JF, Bouma BE, Boppart SA, Fujimoto JG. Optical biopsy in human gastrointestinal tissue using optical coherence tomography. Am J Gastroenterol 1997;92:1800-4.

22. Tearney GJ, Brezinski ME, Southern JF, Bouma BE, Boppart SA, Fujimoto JG. Optical biopsy in human pancreatobiliary tissue using optical coherence tomography. Dig Dis Sci 1998;43:1193-9.

23. Sivak MV Jr, Kobayashi K, Izatt JA, et al. High-resolution endoscopic imaging of the GI tract using optical coherence tomography. Gastrointest Endosc 2000;51:474-9.

24. Jackle S, Gladkova N, Feldchtein F, et al. In vivo endoscopic optical coherence tomography of the human gastrointestinal tract toward optical biopsy. Endoscopy 2000;32:743-9.

25. Poneros JM, Brand S, Bouma BE, Tearney GJ, Compton CC, Nishioka NS. Diagnosis of specialized intestinal metaplasia by optical coherence tomography. Gastroenterology 2001;120:7-12.

26. Zuccaro G, Gladkova N, Vargo J, et al. Optical coherence tomography of the esophagus and proximal stomach in health and disease. Am J Gastroenterol 2001;96:2633-9.

27. Brand S, Poneros JM, Bouma BE, Tearney GJ, Compton CC, Nishioka NS. Optical coherence tomography in the gastrointestinal tract. Endoscopy 2000;32:796-803.

28. Rollins AM, Yazdanfar S, Barton JK, Izatt JA. Real-time in vivo color Doppler optical coherence tomography. J Biomed Optics 2002;7:123-9. 


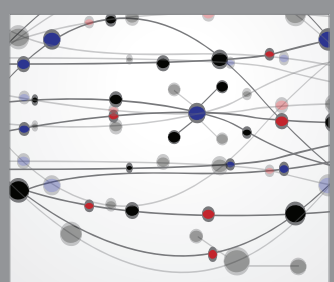

The Scientific World Journal
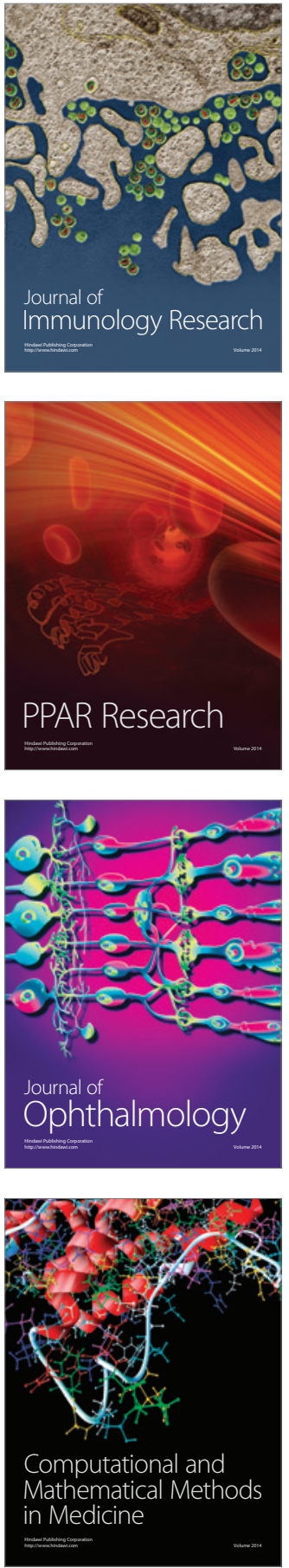

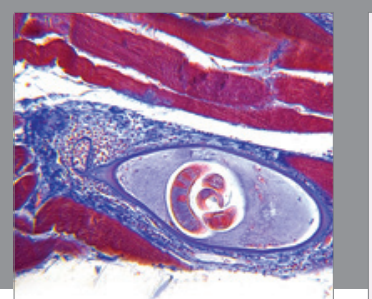

Gastroenterology Research and Practice

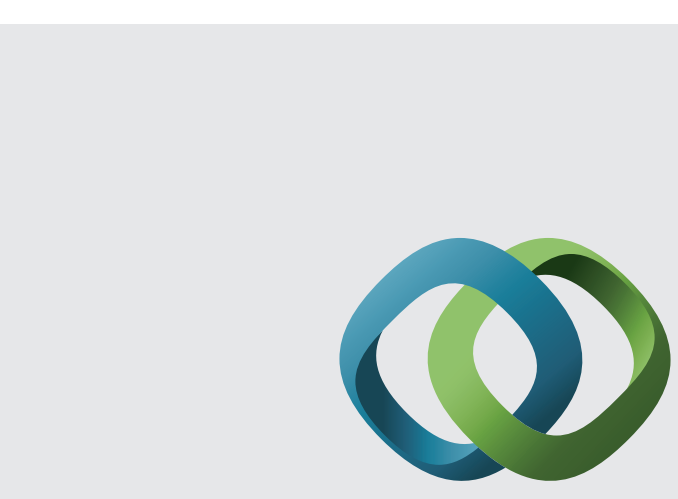

\section{Hindawi}

Submit your manuscripts at

http://www.hindawi.com
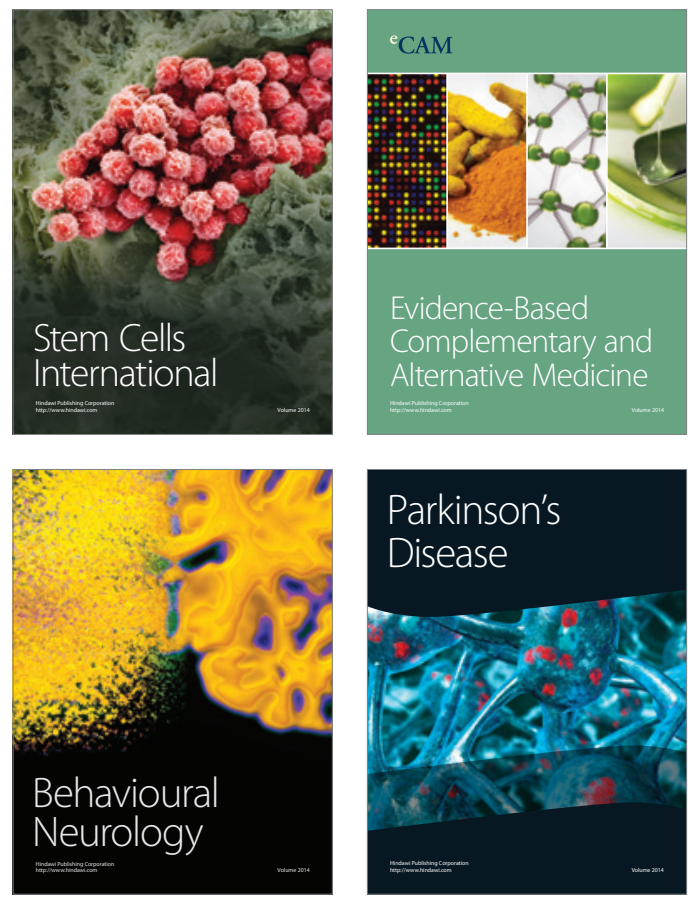
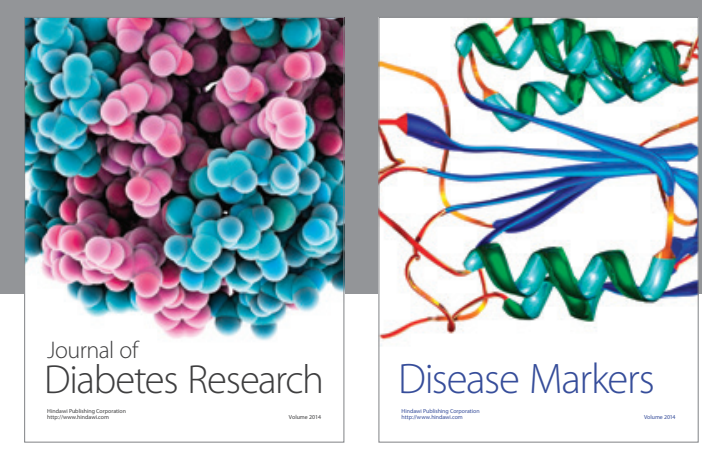

Disease Markers
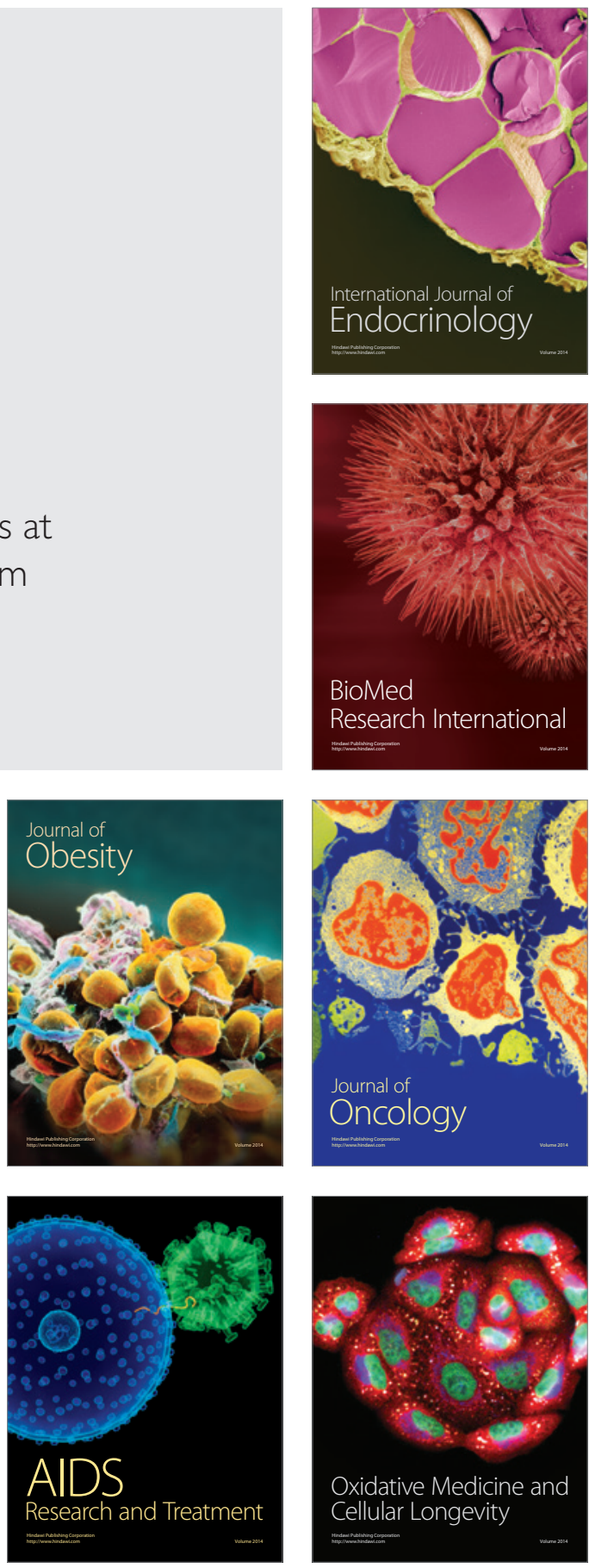